\title{
Cost-effectiveness of combinatorial pharmacogenomic testing for depression from the Canadian public payer perspective
}

\author{
Julie-Anne Tanner ${ }^{1}$ (D), Paige E Davies ${ }^{1}$, Christopher C Overall², Daniel Grima ${ }^{3}$, Julian Nam ${ }^{4}$ \\ \& Bryan M Dechairo*,2 \\ ${ }^{1}$ Assurex Health Ltd, a subsidiary of Myriad Neuroscience, Toronto, ON, M5T 1L8, Canada \\ ${ }^{2}$ Myriad Genetics Inc., Salt Lake City, UT 84108, USA \\ ${ }^{3}$ Cornerstone Research Group Inc., Burlington, ON, L7N 3H8, Canada \\ ${ }^{4}$ Hoffmann-La Roche Ltd, Mississauga, ON, L5N 5M8, Canada \\ *Author for correspondence: Bryan.Dechairo@myriad.com
}

\begin{abstract}
Aim: Evaluate the cost-effectiveness of combinatorial pharmacogenomic (PGx) testing, versus treatment as usual (TAU), to guide treatment for patients with depression, from the Canadian public healthcare system perspective. Materials \& methods: Clinical and economic data associated with depression were extracted from published literature. Clinical (quality-adjusted life years; QALYs) and economic (incremental cost-effectiveness ratio) outcomes were modeled using combinatorial PGx and TAU treatment strategies across a 5-year time horizon. Results: With the combinatorial PGx strategy to guide treatment, patients were projected to gain 0.14-0.19 QALYs versus TAU. Accounting for test price, combinatorial PGx saved CAD $\$ 1,687-\$ 3,056$ versus TAU. Incremental cost-effectiveness ratios ranged from $-\$ 11,861$ to $-\$ 16,124 / Q A L Y$ gained. Conclusion: Combinatorial PGx testing was more efficacious and less costly compared with the TAU for depression.
\end{abstract}

First draft submitted: 23 January 2020; Accepted for publication: 31 March 2020; Published online: 17 April 2020

Keywords: cost $\bullet$ cost-effectiveness $\bullet$ cost saving $\bullet$ depression $\bullet$ genetic testing $\bullet$ ICER $\bullet$ pharmacogenetics $\bullet$ pharmacogenomics $\bullet$ QALY

Depression is a significant health and economic burden in Canada. In addition to the impact of depression on patients' health and quality of life [1], it costs the Canadian economy more than CAD $\$ 32$ billion annually [2]. The cost of depression stems directly from increased healthcare resource utilization and indirectly as a result of increased disability and absenteeism [3,4]. For example, the direct and indirect per-patient costs were 3.5- and three-times higher, respectively, among a cohort of patients with depression compared with a non-depressed control cohort in Manitoba, Canada [4].

A direct approach to reducing costs associated with depression is through improved treatment efficacy, thereby increasing the number of patients who achieve remission from the disease. The primary treatment approach for depression is antidepressant medication; however, more than half of patients do not respond to their first medication trial and even fewer achieve remission [5]. This can result in a process of medication trial-and-error, leading to additional treatment failures or medication side effects. Better treatment approaches are needed in order to increase patients' probability of achieving remission from their depression and decrease the time to such an outcome.

Pharmacogenomic (PGx) testing can be used to improve the treatment of depression. Combinatorial PGx is a third generation of PGx testing where multiple pharmacokinetic and pharmacodynamic gene-drug interactions are weighted and counterbalanced. This approach is distinct from the first-generation single cytochrome P450 gene tests or the second-generation tests of multiple gene-drug interactions and therefore, combinatorial PGx testing merits its own assessment of efficacy and cost-effectiveness. A recent randomized controlled trial of 1,167 patients with major depression in the USA demonstrated that combinatorial PGx-guided treatment results in a $50 \%$ relative increase (15.3 vs $10.1 \%)$ in remission rate compared with treatment as usual (TAU), as well as decreased depressive 


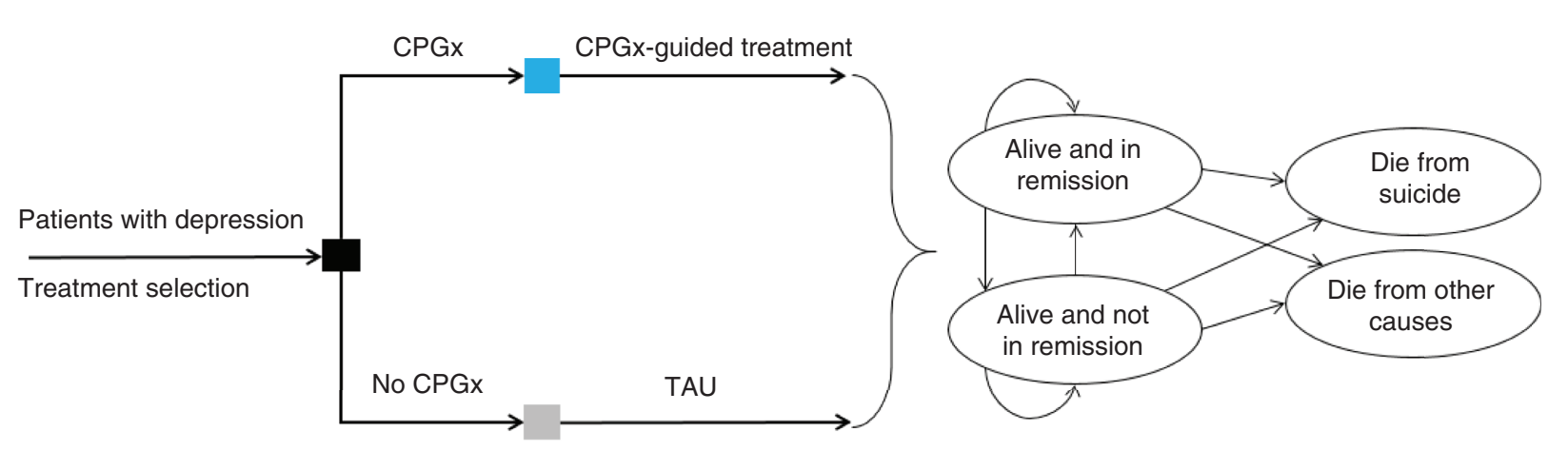

Decision node for test utilization

Decision node for treatment when the patient is tested with GeneSight

Decision node for treatment when the patient is managed as usual

Figure 1. Decision diagram and state-transition model.

CPGx: Combinatorial pharmacogenomic testing; TAU: Treatment as usual.

Decision diagram and state-transition model adapted from [13].

symptoms and greater response rate [6]. These findings are supported by several smaller USA clinical trials [7-9] and in Canada, by the large naturalistic IMPACT study that further demonstrated the clinical utility of the combinatorial PGx testing approach [10].

In addition to improving treatment efficacy, previous studies have demonstrated that combinatorial PGx testing results in significant reimbursement savings, reduction in polypharmacy and healthcare utilization and is costeffective among patients with depression in the USA [9,11-14]. However, as there are substantial differences between the USA and Canadian healthcare systems and corresponding costs [15] and there are no published data on the cost-effectiveness of combinatorial PGx testing to guide depression treatment in Canada, it is important to evaluate this in the Canadian setting.

The purpose of the current study was to evaluate the cost-effectiveness of the use of combinatorial PGx testing, compared with treatment as usual, to guide the treatment of patients with moderate-to-severe depression from the perspective of the Canadian public healthcare system, which is a publicly funded healthcare system, including universal coverage for medically necessary healthcare services.

\section{Materials \& methods \\ Overview}

We have developed a model analyzing the cost-effectiveness of using combinatorial PGx testing to guide the treatment of moderate-to-severe major depression compared with using TAU. The model was based loosely on the model by Hornberger and colleagues [13], with notable modifications to include remission as the target end point for depression instead of the earlier end point, response. The model was also updated to include cycle-based transition probabilities for remission, relapse rates and a hazard ratio to allow differential rates of relapse for patients in each model arm. The modelling approach is described in detail in the sections below. This modeling approach followed guidelines outlined in the Consolidated Health Economic Evaluation Reporting Standards (CHEERS) checklist.

The decision diagram and state-transition probability analysis are depicted in Figure 1. Four states, assessed annually within the time horizon, have been modeled based on a patient's survival and remission from their depression. The duration of each cycle in the model is 1 year. All base-case input parameters, including the probabilities associated with mortality and remission, are provided in Table 1. Four versions of the model were run. Model 1 refers to the base case and models 2-4 refer to scenario analyses. The base-case and three scenario analyses are described in the 'Remission rates' section below. 
Table 1. Base-case values and model input parameters.

\begin{tabular}{|c|c|c|}
\hline Analysis parameters & Value & Ref. \\
\hline \multicolumn{3}{|l|}{ Test characteristics } \\
\hline Remission rate, $\mathrm{TAU}^{\dagger}$ & $12.8 \%, 10.0 \%, 20.3 \%, 10.1 \%$ & [6-9] \\
\hline Remission rate, $\mathrm{CPG} \mathrm{x}^{\dagger}$ & $18.9 \%, 15.1 \%, 28.4 \%, 14.9 \%$ & [6-9] \\
\hline Relative benefit ratio for remission, CPGx compared with $\mathrm{TAU}^{\dagger}$ & $1.53,1.55,1.47,1.51$ & [6-9] \\
\hline Catch-up year (duration of test effect) - remission & 3 & [16] \\
\hline Relapse rate, TAU & $23.3 \%$ & [17] \\
\hline Relapse rate, CPGx & $9.9 \%$ & [17] \\
\hline Hazard ratio for relapse, CPGx compared with TAU & 0.39 & [17] \\
\hline Catch-up year (duration of test effect) - Relapse & 3 & [16] \\
\hline \multicolumn{3}{|l|}{ Clinical parameters } \\
\hline Starting age of patient & 32 years old & [18] \\
\hline Relative risk of all-cause mortality, non-remitters & 2.31 & [4] \\
\hline Relative risk of all-cause mortality, remitters & 1.38 & [4] \\
\hline Suicide mortality rates, non-remitters & $0.04 \%$ & [4] \\
\hline Suicide mortality rates, remitters & $<0.01 \%$ & [4] \\
\hline \multicolumn{3}{|l|}{ Costs $(2018$ CAD $\$)$} \\
\hline CPGx testing & $\$ 2500$ & \\
\hline Annual direct medical costs, remitters & $\$ 2832$ & [4] \\
\hline Annual direct medical costs, nonremitters & $\$ 10,064$ & [4] \\
\hline Annual indirect medical costs, remitters & $\$ 510$ & [4] \\
\hline Annual indirect medical costs, nonremitters & $\$ 1522$ & [4] \\
\hline \multicolumn{3}{|l|}{ Utilities (quality of life) } \\
\hline Remission following therapy & 0.83 & [3] \\
\hline Nonremission following therapy & 0.55 & [3] \\
\hline \multicolumn{3}{|l|}{ Policy parameters } \\
\hline Time horizon, years & 5 & \\
\hline Discount rate & $3 \%$ & \\
\hline \multicolumn{3}{|c|}{$\begin{array}{l}\text { † Four models were run using different remission rates and RBR for CPGx and TAU. Model 1: meta-analysis of all four studies (TAU } 12.5 \%, \text { CPGx } 18.9 \% \text {, } \\
\text { RBR 1.53). Model 2: meta-analysis of CPGx blinded RCTs (TAU 10.0\%, CPGx 15.1\%, RBR 1.55). Model 3: meta-analysis of CPGx open-label studies (TAU } \\
\text { 20.3\%, CPGx 28.4\%, RBR 1.47). Model 4: largest blinded CPGx RCT alone (TAU 10.1\%, CPGx 14.9\%, RBR 1.51). Random effects models were used for all } \\
\text { meta-analyses. } \\
\text { CPGx: Combinatorial pharmacogenomic testing; RBR: Relative benefit ratio; RCT: Randomized controlled trial; TAU: Treatment as usual. }\end{array}$} \\
\hline
\end{tabular}

\section{Study population, treatment \& time horizon}

This analysis focused on patients with moderate-to-severe depression whose antidepressant treatment was guided by combinatorial PGx testing compared with patients whose treatment was unguided, and therefore underwent TAU. The starting age of the patient in this model was 32 years based on the median age of onset for major depressive disorder reported by Kessler $e t$ al. [18]. Due to the chronic and recurrent nature of major depression and in order to capture both early and downstream costs and savings associated with the management of this disease, a lifetime time horizon would be appropriate. However, we selected a conservative time horizon of 5 years, to align with previous analyses by Health Quality Ontario [19].

\section{Remission rates}

In order to quantify remission rates associated with combinatorial PGx-guided treatment and TAU for depression, we conducted meta-analyses of the per-protocol populations of the four published prospective clinical trials of the GeneSight combinatorial PGx testing for depression (Supplementary Figure 1) [6-9]. The methodology used for the meta-analyses was based on the previous meta-analysis of GeneSight conducted by Brown et al. [20], where study selection, PRISMA guidelines and assessment of bias were described in detail. The remission rates for combinatorial PGx and TAU derived from the meta-analysis of all four clinical trials are included in model 1, the base-case model. For the scenario analyses (models 2-4), remission rates were derived as follows. A second meta-analysis (model 2) was performed to determine remission rates reported in blinded randomized controlled trials ( $\mathrm{n}=2$ studies), where 
placebo effect has been accounted for [6,9]. A third meta-analysis of the open-label clinical trials (model 3, $\mathrm{n}=2$ studies) provided an estimate of remission rates in the real world, where placebo effect is not controlled $[7,8]$. Finally, for the last scenario analysis (model 4), we used the remission rates from only one study - the largest $(\mathrm{n}=1167)$ blinded, randomized, controlled trial of combinatorial PGx for depression [6].

The model's output is dependent on both the absolute difference in remission rates between treatment strategies and the relative benefit of one strategy over the other. Therefore, a random effects model was used for all metaanalyses to weight the remission rates from each study and derive the relative risk (RR) associated with combinatorial PGx testing relative to TAU, referred hereafter as the relative benefit ratio (RBR). The combinatorial PGx group remission rate was calculated from the weighted TAU remission rate and the RBR, using the following formula: remission rate with combinatorial pharmacogenomic testing $=1-(1-$ remission rate TAU $) \wedge$ RBR. The formula ensures that the remission rate for the combinatorial PGx strategy cannot exceed $100 \%$ no matter how large the RBR gets. All meta-analyses were conducted using the 'meta' package in R software version 3.5.0 (R Foundation for Statistical Computing, Vienna, Austria). Aside from remission rate and RBR, all other parameters were identical for the base-case scenario of the four models.

It was assumed that the relative benefit of the combinatorial PGx testing strategy over TAU in terms of remission rate would persist for 3 years, as the pharmacological treatment effect over placebo has been reported to persist up to 36 months according to a systematic review of randomized trials of continued antidepressant treatment [16]. Therefore, in our model, after 3 years, the relative benefit of combinatorial PGx testing ended and patients in both arms experience a similar probability of achieving remission.

\section{Relapse rates}

The rate of relapse following remission was determined for the combinatorial PGx group using unpublished data from the Genomics Used to Improve DEpression Decisions (GUIDED) clinical trial (NCT02109939). Of note, the primary outcomes from this trial have been published by Greden $e t$ al. [6]. At 24 weeks following treatment guided by combinatorial PGx testing, 9.1\% of patients relapsed after previously achieving remission from their depression. We were unable to determine the relapse rate at 24 weeks for the TAU arm in the same clinical trial because this group was unblinded at week 12 and therefore was able to use the combinatorial PGx test report to guide treatment for weeks $12-24$ of the study. Instead, our model used the TAU relapse rate of $23.3 \%$, reported in a systematic review of antidepressant clinical trials by Sim et al. [17]. A hazard ratio (HR) of 0.39 was calculated from the combinatorial PGx and TAU relapse rates. The relapse rate for the combinatorial PGx strategy was then adjusted using the following formula to ensure that the rate remains between 0 and 1 regardless of the HR: relapse rate with combinatorial pharmacogenomic testing $=1-(1-$ relapse rate TAU $) \wedge \mathrm{HR}$. This resulted in a relapse rate of $9.9 \%$ in the combinatorial PGx group. The reduced relapse rate in the combinatorial PGx compared with TAU group was projected to last 3 years. Like remission rates, after 3 years the relapse rates in both arms of the model became equal.

\section{Mortality rates}

Mortality rates were estimated from a Canadian study of the clinical and economic burden of depression [4]. The study compared clinical outcomes, healthcare utilization, direct costs and social services costs between a depression and matched-control cohort. For the purpose of our model, the control and depression cohorts served as surrogates for remitters and nonremitters, respectively. Annual suicide mortality rates were $<0.01$ and $0.04 \%$ for remitters and nonremitters, respectively [4]. To estimate the impact of the treatment arm on all-cause mortality risk, the RR was calculated for remitters and nonremitters compared with the general Canadian population [4,21]. The RR of all-cause mortality for remitters and nonremitters was 1.37 and 2.29 , respectively.

\section{Costs}

The direct and indirect costs included in the analysis were derived from the same Canadian study of the clinical and economic burden of depression [4]. Direct costs consisted of annual hospital, physician and prescription drug costs. Indirect, or social services, costs consisted of annual rent assistance and employment income assistance. In this model, direct and indirect costs accumulated over a patient's lifetime, within the time horizon, whereas the $\$ 2500$ combinatorial PGx test price was applied as a one-time cost for that treatment strategy. All costs are reported in 2018 Canadian dollars. 


\begin{tabular}{|c|c|c|c|c|c|c|c|c|c|}
\hline \multirow[t]{2}{*}{ Model } & \multirow[t]{2}{*}{ Model type } & \multicolumn{2}{|c|}{ Remission rate } & \multirow{2}{*}{$\begin{array}{l}\text { Relative } \\
\text { benefit ratio }\end{array}$} & \multirow{2}{*}{$\begin{array}{l}\text { QALYs } \\
\text { gained }\end{array}$} & \multirow{2}{*}{$\begin{array}{l}\text { Difference in } \\
\text { total costs } \ddagger\end{array}$} & \multirow{2}{*}{$\begin{array}{l}\text { ICER (incremental cost/QALY } \\
\text { gained) } \ddagger\end{array}$} & \multirow[t]{2}{*}{ Conclusion } & \multirow[t]{2}{*}{ Ref. } \\
\hline & & $\mathrm{CPGx}^{\dagger}$ & TAU & & & & & & \\
\hline 1 & $\begin{array}{l}\text { Meta-analysis of all four } \\
\text { CPGx studies }\end{array}$ & $18.9 \%$ & $12.8 \%$ & 1.53 & 0.168 & $-\$ 2431$ & $-\$ 14,454 / \mathrm{QALY}$ & $\begin{array}{l}\text { Dominant, } \\
\text { cost-saving }\end{array}$ & {$[6,7,8,9]$} \\
\hline 2 & $\begin{array}{l}\text { Meta-analysis of CPGx } \\
\text { blinded RCTs }\end{array}$ & $15.1 \%$ & $10.0 \%$ & 1.55 & 0.149 & $-\$ 1878$ & $-\$ 12,580 / \mathrm{QALY}$ & $\begin{array}{l}\text { Dominant, } \\
\text { cost-saving }\end{array}$ & {$[6,9]$} \\
\hline 3 & $\begin{array}{l}\text { Meta-analysis of CPGx } \\
\text { open-label studies }\end{array}$ & $28.4 \%$ & $20.3 \%$ & 1.47 & 0.190 & $-\$ 3056$ & $-\$ 16,124 / \mathrm{QALY}$ & $\begin{array}{l}\text { Dominant, } \\
\text { cost-saving }\end{array}$ & {$[7,8]$} \\
\hline 4 & $\begin{array}{l}\text { Largest blinded CPGx RCT } \\
\text { alone }\end{array}$ & $14.9 \%$ & $10.1 \%$ & 1.51 & 0.143 & $-\$ 1687$ & $-\$ 11,816 /$ QALY & $\begin{array}{l}\text { Dominant, } \\
\text { cost-saving }\end{array}$ & [6] \\
\hline
\end{tabular}

\section{Utilities}

Quality-adjusted life years (QALYs), a standardized measure of the quality and quantity of life lived used to quantify the value of the intervention, were derived from utility values for patients with major depression who are in remission (0.83) compared with the baseline score for patients with major depression (0.55), representing patients who are not in remission [3]. Utility scores can range from 0 to 1 , signifying the health states of death to perfect health. The QALYs were calculated as the utility-weighted sum of the probability of being in remission or not in remission for each treatment strategy, across the 5-year time horizon. A discount rate of $3 \%$ was applied to all costs and health outcomes.

QALYs and incremental costs are used to calculate the incremental cost-effectiveness ratio (ICER), which is a standardized measure used to evaluate the economic value of one intervention over another. The ICER is equal to the incremental cost divided by QALYs.

\section{Sensitivity analyses}

One-way sensitivity analyses were performed separately for all four models. All base-case parameters were varied individually to determine their influence on incremental cost difference and ICER for the combinatorial PGx strategy compared with TAU. The range was set to $\pm 25 \%$ of the mean for all parameters except for those measured in years, where the range was \pm 2 full years. A probabilistic sensitivity analysis was also performed for each of the four models using PyMC3, a probabilistic programming module for Python (PyMC3 version 3.7; Python version 3.7.3). Realistic probability distributions were defined for each model parameter (Supplementary Table 2) and simulations were run based on the parameter values sampled from the underlying distribution using Markov chain Monte Carlo sampling. In this study, 10,000 simulations were performed for each of the four models and outcome metrics were assessed across the ensemble of simulations.

\section{Results}

\section{Base-case \& scenario analyses}

The results of the four models are provided in Table 2. In the base-case model, the combinatorial PGx strategy was associated with a 1.53-times greater remission rate and patients were projected to gain 0.17 QALYs (2.02 months), relative to the TAU strategy. After accounting for the price of the test, the combinatorial PGx strategy saved $\$ 2431$ compared with TAU. The negative ICER demonstrated that the combinatorial PGx treatment strategy was dominant over the TAU strategy, as it was both more efficacious and cost-saving in all scenarios.

The same trends were observed in the scenario analyses (models 2-4). The relative benefit of the combinatorial PGx treatment strategy ranged from $1.47-1.55$, resulting in a projected gain of $0.14-0.19$ QALYs (1.722.28 months) relative to the TAU strategy. After accounting for the price of the test, the combinatorial PGx strategy saved \$1687-3056 compared with TAU. According to the ICERs for the three models, the combinatorial PGx treatment strategy remained dominant over the TAU strategy.

\section{Sensitivity analyses}

One-way sensitivity analyses were performed separately for the four models (Figure 2; Supplementary Figure 2 


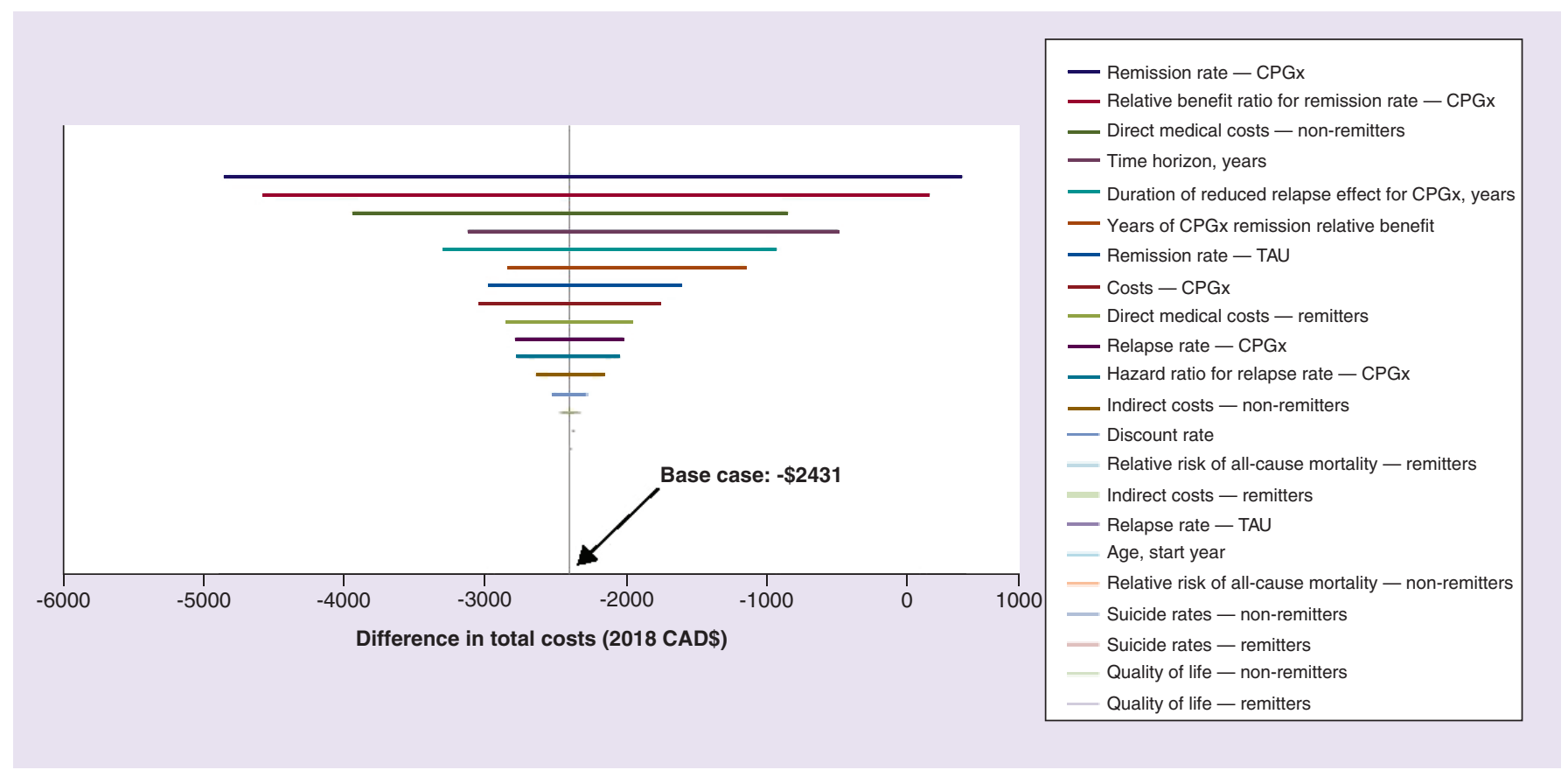

Figure 2. Tornado diagram for difference in total costs between combinatorial pharmacogenomic testing and treatment as usual based on one-way sensitivity analysis. Negative values indicate lower costs in the CPGx relative to TAU group. The cost of the test was included. Analysis refers to model 1, the meta-analysis of all four CPGx studies.

CPGx: Combinatorial pharmacogenomic testing; TAU: Treatment as usual.

\& Supplementary Table 1). In all models, the incremental costs and ICERs were most sensitive to changes in the remission rate in the combinatorial PGx group and second, to variation in the RBR of the combinatorial PGx treatment strategy over TAU. In models 1, 2 and 4, the combinatorial PGx strategy was dominant over the TAU strategy and the ICER remained cost-saving (negative cost per QALY gained) when varying more than $90 \%$ of the input parameters (20/22 parameters). The two scenarios where the combinatorial PGx strategy was more costly, but still more effective, than the TAU strategy were when the combinatorial PGx remission rate and the RBR took their lower limits. For model 1, this resulted in incremental costs for the combinatorial PGx strategy compared with the TAU strategy of $\$ 284$ when remission rate took the lower limit and $\$ 64$ when the RBR took the lower limit. For model 2, the combinatorial PGx incremental costs were $\$ 490$ and 356, respectively, when remission rate and RBR took the lower limit. For model 4 , the incremental costs for the same scenarios were $\$ 649$ when remission rate took the lower limit and $\$ 527$ when the RBR took the lower limit. In model 3, the combinatorial PGx strategy was dominant over the TAU strategy when varying more than $95 \%$ of the parameters (21/22 parameters). In the one higher cost scenario, the incremental cost of the combinatorial PGx strategy relative to TAU was $\$ 124$. This occurred when remission rate in the combinatorial PGx group took the lower limit. In all four models, The ICERs remained below the willingness to pay (WTP) threshold of $\$ 50,000 /$ QALY when varying all input parameters.

These sensitivity analyses were limited to a range of 2 to 7 years for the time horizon. However, should a lifetime time horizon of 50 years be employed in our model, the difference in QALYs, cost savings and ICERs change by up to 32, 66 and 26\%, respectively, where combinatorial PGx further dominates the TAU strategy.

A probabilistic sensitivity analysis was run for the four models (Figure 3; Supplementary Figure 3 \& Supplementary Table 2). The difference in total costs, or incremental cost, and the difference in QALYs are depicted using scatter plots. The northwest, northeast, southwest and southeast quadrants of the scatter plots represent the four planes of cost-effectiveness, indicating neither effective nor cost-saving, effective but not cost-saving, not effective but cost-saving and both effective and cost-saving, respectfully. In all models, the combinatorial PGx treatment strategy dominated the TAU strategy for the majority of simulations (70.3-93.0\%). That is, the combinatorial PGx treatment strategy was both more effective and cost-saving (southeast quadrant) than the TAU strategy. The probability of combinatorial PGx treatment being cost-effective at the WTP threshold of $\$ 50,000$ for all four models is $94.5-96.7 \%$. 


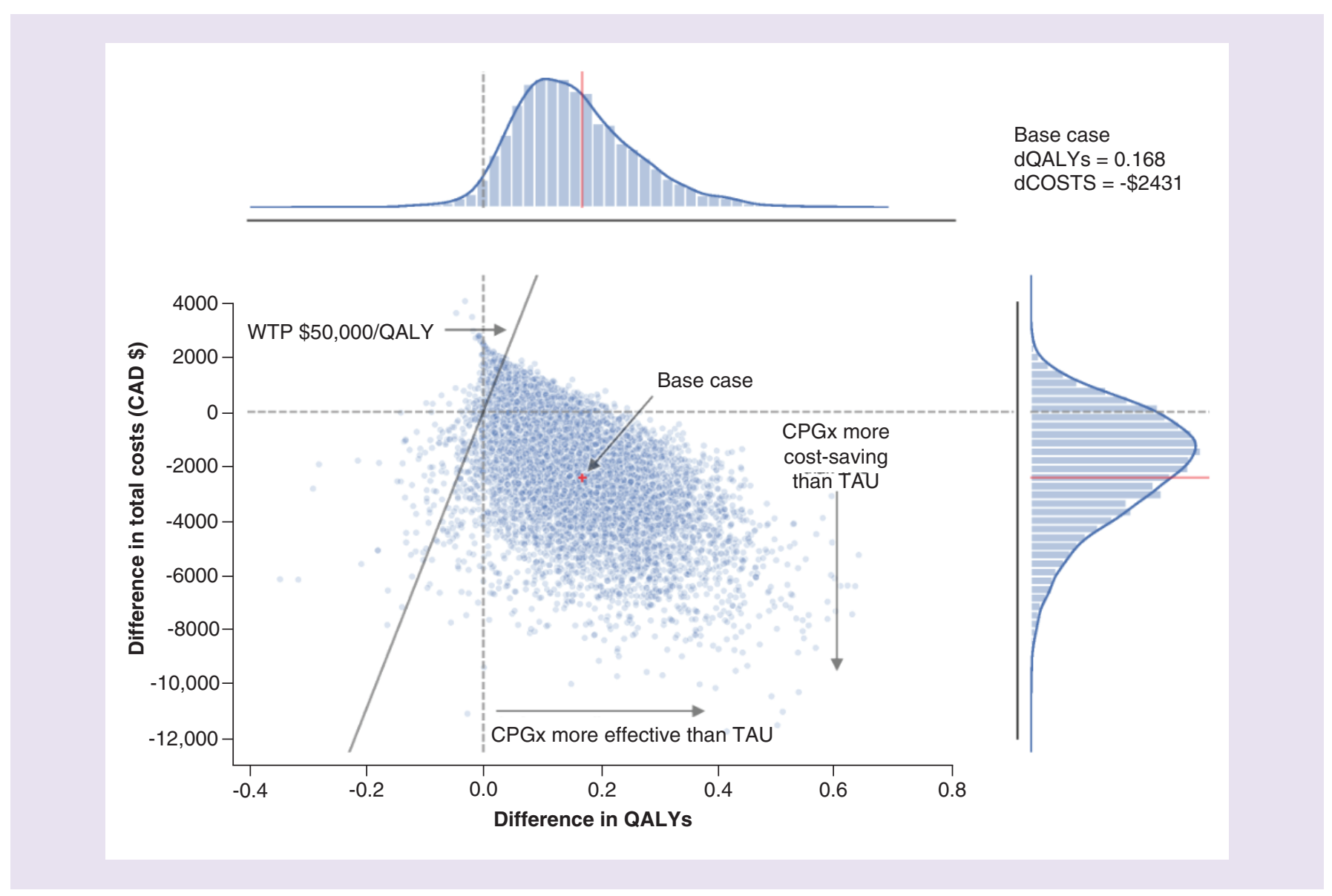

Figure 3. Scatter plot of difference in total costs and difference in effects estimated through probabilistic sensitivity analysis. Probabilistic sensitivity analysis simulations are represented by blue points. The diagrams in the margins represent the univariate distributions. The red line indicates the base case value and the grey line indicates zero. Analysis refers to model 1, the meta-analysis of all four CPGx studies.

CPGx: Combinatorial pharmacogenomic testing; dCOST: Difference in total cost; dQALY: Difference in quality adjusted life year; QALY: Quality adjusted life year; TAU: Treatment as usual; WTP: Willingness to pay.

\section{Discussion}

In the base-case model and scenario analyses, combinatorial PGx dominated the TAU strategy, indicating that it is not only more efficacious but also cost-saving, supporting use in clinical practice. The ICERs derived from the base case model and scenario analyses remained well below the common cost-effectiveness threshold of $\$ 50,000$ when varying any of the parameters in the one-way sensitivity analyses. The parameter that had the greatest impact on the model when varied in one-way sensitivity analyses was the remission rate associated with combinatorial PGx-guided treatment. When remission rate took the lower limit, the combinatorial PGx strategy was associated with \$124-649 higher costs than the TAU strategy, while ICERs remained well below $\$ 50,000$. Based on the probabilistic sensitivity analysis for all four models, there was greater than $94 \%$ probability of combinatorial PGx being cost effective within the WTP threshold of $\$ 50,000$, indicating the robustness of this model. Furthermore, the relative improvement in remission rates for the combinatorial PGx treatment approach compared with TAU is consistent across the different types of studies, despite the differences in remission rates between the RCT and open-label studies. All four models demonstrated a similar $50 \%$ improvement in remission rate for combinatorial PGx compared with TAU, strengthening the validity of the base-case model and the remission rates used therein.

In order to put these findings into perspective, the cost-effectiveness of combinatorial PGx-guided treatment could be considered in relation to other treatment approaches for depression that are available in Canada. For example, Health Quality Ontario, a provincial organization that conducts health technology assessments and provides public funding recommendations to the Ontario Ministry of Health and Long-Term Care, has published 
cost-effectiveness analyses of repetitive transcranial magnetic stimulation, internet cognitive behavioral therapy and group and individual psychotherapy for depression, compared with standard care $[19,22,23]$. These models produced ICERs ranging from $\$ 3,715$ to $\$ 98,242$ per QALY and all three treatment approaches were given positive funding recommendations for use when patients with depression met prespecified criteria. In comparison, the combinatorial PGx-guided treatment approach yielded significantly lower ICERs ranging from $-\$ 11,861$ to $-\$ 16,124$ per QALY gained, with the probability of cost-effectiveness ranging from $94.5-96.7 \%$ at a WTP threshold of $\$ 50,000$ per QALY gained or $89.4-96.6 \%$ at a WTP threshold of $\$ 20,000$ per QALY gained. These results are one piece of evidence in which combinatorial PGx testing to guide depression treatment demonstrates cost savings for the public payer.

Furthermore, emphasis should be placed on novel treatment approaches that are efficacious in both primary care and psychiatric care settings. In Canada, the number of practicing psychiatrists is decreasing and the unmet need for psychiatric services is increasing [24]. A Canadian study of the use of combinatorial PGx to guide depression treatment among primary care providers and psychiatrists demonstrated clinical utility in both physician groups, with the greatest improvement among patients whose treatment was provided by their primary care provider [10]. Optimizing the treatment of depression in primary care could increase psychiatrists' capacity to focus on the most severely ill patients. Therefore, given that the combinatorial PGx treatment strategy is effective in multiple treatment settings and is projected to be cost saving for provincial governments, this may be one approach to reduce wait times for specialists in Canada.

Another cost-effectiveness analysis [25] has demonstrated the cost-effectiveness of PGx testing among $\mathrm{n}=261$ patients with moderate-to-severe depression. As the current study modeled the impact of combinatorial PGx on $\mathrm{n}=1,425$ patients with depression achieving remission, the results cannot be directly compared with the model by Groessl et al., where $\mathrm{n}=261$ patients were included and the target end point was response.

There were several limitations to the modeling approach used in this cost-effectiveness analysis. First, as longterm economic data associated with combinatorial PGx testing for depression were not available in the Canadian healthcare setting, the current study used an indirect assessment of costs associated with combinatorial PGx-guided depression treatment compared with TAU. We applied remission rates from the literature to Canadian costs associated with depression from the literature in order to derive costs associated with each treatment strategy. Moreover, the depression-related costs used in the model were mainly direct costs associated with the treatment of depression (hospital, physician and prescription drug costs), whereas indirect costs were limited to select social services (rent assistance and employment income assistance). Additional indirect costs that could be considered in future cost-effectiveness modeling include disability, absenteeism and lost productivity associated with depression, which are associated with a significant burden on Canada's economy of approximately $\$ 32$ billion annually [2].

Furthermore, this study is limited by assumptions made when empirical data were not available. For example, where long-term durability data were unavailable, we projected a 3-year relative benefit of combinatorial PGx over TAU. This was based on the duration of benefit of pharmacotherapy [16] and mirrored the previously published US cost-effectiveness model of combinatorial PGx for depression [13].

This study may also be limited by the use of a 5 -year time horizon, which may underestimate the long-term costsavings associated with combinatorial PGx-guided treatment of depression. Both the National Institute for Health and Care Excellence (NICE) and the Canadian Agency for Drugs and Technologies in Health (CADTH) guidelines for economic evaluations recommend a time horizon that is long enough to capture all important differences in costs or outcomes between the two treatment strategies [26,27]. In addition, when a treatment strategy may influence mortality, the recommended time horizon is the patient's lifetime [28]. When using a lifetime time horizon of 50 years in an exploratory analysis within the current model, the difference in QALYs, cost savings and ICERs changed by up to 32,66 and 26\%, respectively, where combinatorial PGx further dominates the TAU strategy. However, using the lifetime perspective can create analytical challenges where highly uncertain extrapolations are used. Therefore, for the purpose of this cost-effectiveness assessment, we selected a conservative 5-year time horizon for our primary analysis, as indicated in a recent Health Technology Assessment of psychotherapy for depression and anxiety by Health Quality Ontario [19].

Additionally, we conservatively selected a discount rate of $3 \%$, instead of the $1.5 \%$ discount rate suggested by NICE and the CADTH for economic analyses [27]. However, when the model was re-run with the $1.5 \%$ discount rate, there was little impact on the model output and the incremental costs, QALYs and ICERs further favored combinatorial PGx over TAU. 
At last, the relapse rates associated with each treatment strategy used in the model were derived from different studies. The relapse rate for patients using the combinatorial PGx treatment strategy was derived from unpublished data from the GUIDED clinical trial, whereas relapse for patients using the TAU strategy was reported in a systematic review of antidepressant clinical trials by $\operatorname{Sim} e t$ al. [17]. As there will inevitably be differences in study design, the relapse rates are simply estimates for the two treatment strategies and sensitivity analyses are particularly important in this situation. When relapse rates were varied in one-way sensitivity analyses, the resulting incremental costs and the ICERs remained lower than $-\$ 10,000$ (i.e., cost saving). Extending this further, when the combinatorial PGx relapse rate was increased by $100 \%$, all ICERs remained below $-\$ 5000$, signifying that even at the upper limit of relapse, the model remains cost saving.

\section{Conclusion}

The present study examined the cost-effectiveness of using combinatorial PGx testing to guide the treatment of depression compared with treatment as usual. The combinatorial PGx treatment strategy was more efficacious (gain in QALYs) and less costly (negative incremental costs) compared with the TAU strategy for depression. With the support of one-way and probabilistic sensitivity analyses, we conclude that combinatorial PGx testing is costeffective compared with TAU for patients with moderate-to-severe depression from the perspective of the Canadian public healthcare system. Considering that approximately 1.6 million Canadians have reported unmet or only partially met mental healthcare needs [29], our approach for treating mental illness, including depression, requires improvement and greater investment. However, in Canada, mental health accounts for $7.2 \%$ of total government health expenditures, which, in the international context, is less than most developed countries [30]. According to this cost-effectiveness analysis, investing in the use of combinatorial PGx testing to guide the treatment of depression is projected to improve patient outcomes and quality of life, as well as provide cost-savings for the Canadian public healthcare system.

\section{Summary points}

- Better treatment approaches for depression are needed in order to increase patients probability of achieving remission and decrease the time to such an outcome. One approach that can be used to improve depression treatment is pharmacogenomic testing.

- Combinatorial pharmacogenetic testing is a third generation of pharmacogenetic testing where multiple pharmacokinetic and pharmacodynamic gene-drug interactions are weighted and counterbalanced. This approach is distinct from the first-generation single-gene tests or the second-generation tests of multiple gene-drug interactions and therefore, merits its own assessment of efficacy and economic utility.

- Determining the cost-effectiveness of new healthcare technologies is vital for decision makers and for implementation of the technology.

- From the Canadian public payer perspective, we assessed the cost-effectiveness of using combinatorial pharmacogenetic testing to guide the treatment of depression.

- Using combinatorial pharmacogenetic testing to guide depression treatment, patients are projected to gain 0.14-0.19 quality-adjusted life years (approximately 2 months) compared with using treatment as usual, across a 5-year time period.

- Combinatorial pharmacogenetic testing is projected saved the public payer CAD \$1687-3056 compared with treatment as usual, over 5 years.

- Combinatorial pharmacogenetic testing is both cost saving and cost-effective, with projected incremental cost-effectiveness ratios ranging from $-\$ 11,861$ to $-\$ 16,124$ /quality adjusted life year gains.

- These findings add to the body of evidence demonstrating that combinatorial pharmacogenetic testing is more efficacious and less costly compared with treatment as usual for depression.

Supplementary data

To view the supplementary data that accompany this paper please visit the journal website at: www.futuremedicine.com/doi/suppl/10.2217/pgs-2020-0012

Financial \& competing interests disclosure

This study was fully funded by Assurex Health (now Myriad Neuroscience). Funding during manuscript preparation was supported by Assurex Health and a Mitacs Elevate Postdoctoral Fellowship (JA Tanner). JA Tanner, PE Davies, CC Overall and BM Dechairo were employed by Assurex Health/Myriad at the time of the study. The authors have no other relevant affiliations or financial 
involvement with any organization or entity with a financial interest in or financial conflict with the subject matter or materials discussed in the manuscript apart from those disclosed.

No writing assistance was utilized in the production of this manuscript.

\section{Open access}

This work is licensed under the Attribution-NonCommercial-NoDerivatives 4.0 Unported License. To view a copy of this license, visit http://creativecommons.org/licenses/by-nc-nd/4.0/

\section{References}

Papers of special note have been highlighted as: $\bullet$ of interest; $\bullet \bullet$ of considerable interest

1. Steensma C, Loukine L, Orpana $\mathrm{H}$ et al. Describing the population health burden of depression: health-adjusted life expectancy by depression status in Canada. Health Promot. Chronic Dis. Prev. Can. 36(10), 205-213 (2016).

2. Stonebridge C, Sutherland G. Healthy brains at work. estimating the impact of workplace mental health benefits and programs (2016). www.conferenceboard.ca/temp/62991e2c-3a36-40ea-add2-2332520fc891/8242_Healthy-Brains-Workplace_BR.pdf

3. Mrazek DA, Hornberger JC, Altar CA, Degtiar I. A review of the clinical, economic and societal burden of treatment-resistant depression: 1996-2013. Psychiatr. Serv. 65(8), 977-987 (2014).

- Provides a thorough review of the economic burden of depression in USA, demonstrating the need for better treatments for depression.

4. Tanner J-A, Hensel J, Davies PE, Brown LC, Dechairo BM, Mulsant BH. Economic burden of depression and associated healthcare resource use in Manitoba, Canada. Can. J. Psych. doi:10.1177/0706743719895342 (2019).

- Provides an estimate of the economic burden of depression in Canada, demonstrating the significant burden on the healthcare system and the need for better treatments for depression.

5. Rush AJ, Trivedi MH, Wisniewski SR et al. Acute and longer-term outcomes in depressed outpatients requiring one or several treatment steps: a STAR*D report. Am. J. Psychiatry 163(11), 1905-1917 (2006).

6. Greden JF, Parikh SV, Rothschild AJ et al. Impact of pharmacogenomics on clinical outcomes in major depressive disorder in the GUIDED trial: a large, patient- and rater-blinded, randomized, controlled study. J. Psychiatr. Res. 111, 59-67 (2019).

-. The largest randomized controlled trial (RCT) of combinatorial pharmacogenetic testing to guide depression treatment, reporting the clinical utility and validity of this technology.

7. Hall-Flavin DK, Winner JG, Allen JD et al. Utility of integrated pharmacogenomic testing to support the treatment of major depressive disorder in a psychiatric outpatient setting. Pharmacogenet. Genomics 23(10), 535-548 (2013).

-• One of the early clinical studies of combinatorial pharmacogenetic testing to guide depression treatment.

8. Hall-Flavin DK, Winner JG, Allen JD et al. Using a pharmacogenomic algorithm to guide the treatment of depression. Transl. Psychiatry 2, e172 (2012).

-• One of the early clinical studies of combinatorial pharmacogenetic testing to guide depression treatment.

9. Winner JG, Carhart JM, Altar CA, Allen JD, Dechairo B. A prospective, randomized, double-blind study assessing the clinical impact of integrated pharmacogenomic testing for major depressive disorder. Discov. Med. 16, 219-227 (2013).

-• One of the early clinical studies of combinatorial pharmacogenetic testing to guide depression treatment.

10. Tanner JA, Davies PE, Voudouris NC et al. Combinatorial pharmacogenomics and improved patient outcomes in depression: treatment by primary care physicians or psychiatrists. J. Psychiatr. Res. 104, 157-162 (2018).

11. Benitez J, Cool CL, Scotti DJ. Use of combinatorial pharmacogenomic guidance in treating psychiatric disorders. Per. Med. 15(6), 481-494 (2018).

12. Brown LC, Lorenz RA, Li J, Dechairo BM. Economic utility: combinatorial pharmacogenomics and medication cost savings for mental health care in a primary care setting. Clin. Ther. 39(3), 592-602.e591 (2017).

13. Hornberger J, Li Q, Quinn B. Cost-effectiveness of combinatorial pharmacogenomic testing for treatment-resistant major depressive disorder patients. Am. J. Manag. Care 21(6), e357-e365 (2015).

-. The first cost-effectiveness model of using combinatorial pharmacogenomic testing to guide depression treatment, projected for the US healthcare system.

14. Winner JG, Carhart JM, Altar CA et al. Combinatorial pharmacogenomic guidance for psychiatric medications reduces overall pharmacy costs in a 1 year prospective evaluation. Curr. Med. Res. Opin. 31(9), 1633-1643 (2015).

15. Ottawa ON. Canadian Institute for Health Information. National Health Expenditure Trends, 1975 to 2018 (2018). https://secure.cihi.ca/free_products/NHEX-trends-narrative-report-2018-en-web.pdf

16. Geddes JR, Carney SM, Davies C et al. Relapse prevention with antidepressant drug treatment in depressive disorders: a systematic review. Lancet 361(9358), 653-661 (2003). 
17. Sim K, Lau WK, Sim J, Sum MY, Baldessarini RJ. Prevention of relapse and recurrence in adults with major depressive disorder: systematic review and meta-analyses of controlled trials. Int. J. Neuropsychopharmacol. 19(2), 1-13 (2015).

18. Kessler RC, Berglund P, Demler O, Jin R, Merikangas KR, Walters EE. Lifetime prevalence and age-of-onset distributions of DSM-IV disorders in the National Comorbidity Survey Replication. Arch. Gen. Psychiatry 62(6), 593-602 (2005).

19. Health Quality Ontario. Psychotherapy for major depressive disorder and generalized anxiety disorder: a health technology assessment. Ont. Health Technol. Assess. Ser. 17(15), 1-167 (2017).

- A health technology assessment by Health Quality Ontario of a depression treatment approach, including cost-effectiveness modeling.

20. Brown L, Vranjkovic O, Li J et al. The clinical utility of combinatorial pharmacogenetic testing for patients with depression: a meta-analysis under consideration. Pharmacogenomics doi:10.2217/pgs-2019-0157 (2020).

21. Statistics Canada. Table 13-10-0710-01 Deaths and mortality rates, by age group (2013-2017) https://doi.org/10.25318/1310071001-eng

22. Health Quality Ontario. Repetitive transcranial magnetic stimulation for treatment-resistant depression: an economic analysis. Ont. Health Technol. Assess. Ser. 16(6), 1-51 (2016).

- A health technology assessment by Health Quality Ontario of a depression treatment approach, including cost-effectiveness modeling.

23. Health Quality Ontario. Internet-delivered cognitive behavioural therapy for major depression and anxiety disorders: a health technology assessment. Ont. Health Technol. Assess. Ser. 19(6), 1-199 (2019).

- A health technology assessment by Health Quality Ontario of a depression treatment approach, including cost-effectiveness modeling.

24. Canadian Collaborative Centre for Physician Resources. Psychiatry - a recent profile of the profession. Canadian Medical Association (2012). http://nationalphysiciansurvey.ca/wp-content/uploads/2012/10/C3PR-Bulletin-201204-EN.pdf

25. Groessl EJ, Tally SR, Hillery N, Maciel A, Garces JA. Cost-effectiveness of a pharmacogenetic test to guide treatment for major depressive disorder. J. Manag. Care Spec. Pharm. 24(8), 726-734 (2018).

26. National Institute for Health and Care Excellence (NICE). Guide to the methods of technology appraisal 2013. (2013). www.ncbi.nlm.nih.gov/books/NBK395867/pdf/Bookshelf_NBK395867.pdf

27. Guidelines for the Economic Evaluation of Health Technologies: Canada (4th Edition). (2017). www.cadth.ca/sites/default/files/pdf/guideli nes_for_the_economic_evaluation_of_health_technologies_canada_4th_ed.pdf

28. Cohen DJ, Reynolds MR. Interpreting the results of cost-effectiveness studies. J. Am. Coll. Cardiol. 52(25), 2119-2126 (2008).

29. Sunderland A, Findlay LC. Perceived need for mental health care in Canada: results from the 2012 Canadian Community Health Survey-Mental Health. Health Rep. 24(9), 3-9 (2013).

30. Jacobs P, Dewa C, Lesage A et al. The cost of mental health and substance abuse services in Canada (2010). www.ihe.ca/publications/the-cost-of-mental-health-and-substance-abuse-services-in-canada 
